\section{A peculiar case of autoimmune pancreatitis and sclerosing cholangitis}

A 65-year-old man presented with recurrent abdominal pain and slight weight loss. Computed tomography (CT) and endoscopic ultrasound (EUS) revealed a hypodense, partially cystic mass located in the pancreatic body and tail that raised the suspicion of pancreatic cancer ( $\bullet$ Fig. 1). No distant metastases were detected and the patient underwent surgical resection of the mass and splenectomy, which was complicated by a bleeding splenic artery aneurysm. Surprisingly, only massive inflammation of the pancreas was found on histological examination of the resected specimen.

The patient re-presented 1 year later with jaundice for the first time. EUS demonstrated a pseudocyst within the head of the pancreas. Endoscopic retrograde cholangiopancreatography (ERCP) revealed an isolated stenosis of the distal common bile duct ( Fig. 2a). This was successfully treated with regularly exchanged endoprotheses, leading to a complete normalization of laboratory parameters.

The patient returned 2 years later with fever and jaundice. This time ERCP showed a purulent cholangitis with a stricture of the biliary bifurcation as well as several stenotic intrahepatic bile ducts ( Fig. 2 b). Brush cytology raised a suspicion of cholangiocarcinoma. Serum IgG4 levels, antinuclear antibody (ANA), antineutrophil cytoplasmic antibody (ANCA), anticarbonic anhydrase II and antilactoferrin antibodies were all within the nor- mal range; however, there was peripheral eosinophilia, which can be associated with autoimmune pancreatitis (AIP) [1]. Histological re-examination of the surgical specimen revealed an impressive lymphoplasmacytic infiltration of the resected pancreas with IgG4-positive cells (- Fig.3a,b). This led finally to the correct diagnosis of AIP type 1 with metachronous autoimmune cholangitis. The patient was treated according to the recently published diagnostic algorithms [2-4] with long-term, slowly tapered prednisone, with resulting improvement in his clinical symptoms, biliary strictures (๑ Fig.2c), blood eosinophilia, and laboratory parameters.

In summary, distinguishing AIP from pancreatic cancer remains a pitfall for clinicians; in some cases, surgical resection remains the treatment of choice because IgG4 levels can be elevated in 10\% of pancreatic cancers [5].

Endoscopy_UCTN_Code_CCL_1AZ_2AG

Competing interests: None

\section{H.-M. Curth ${ }^{1}$, U. Drebber ${ }^{2}$, D. Stippel ${ }^{3}$, C. Bangard ${ }^{4}$, U. Töx ${ }^{1}$, H.-M. Steffen ${ }^{1}$}

' Department of Gastroenterology and Hepatology, University of Cologne, Germany

${ }^{2}$ Institute of Pathology, University of Cologne, Germany

3 Department of General, Visceral and Cancer Surgery, University of Cologne, Germany

${ }^{4}$ Department of Radiology, University of Cologne, Germany
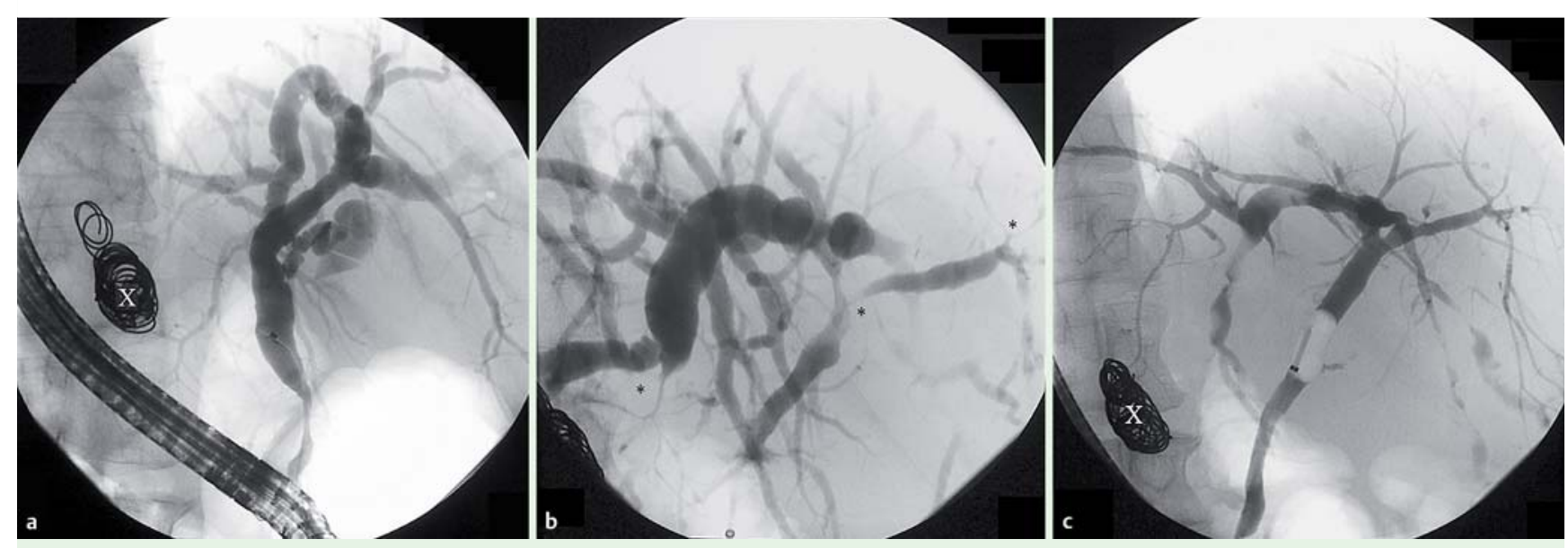

Fig. 2 Images during endoscopic retrograde cholangiopancreatography (ERCP). a A stenosis of the distal common bile duct is shown 1 year after surgical resection of the pancreatic mass. $\mathbf{b}$ A hilar stenosis and several intrahepatic bile duct stenoses $\left(^{*}\right)$ are seen 2 years later. $\mathbf{c}$ Significant improvement of the stenoses is seen following corticosteroid therapy. (X indicates coils within a splenic artery aneurysm.) 


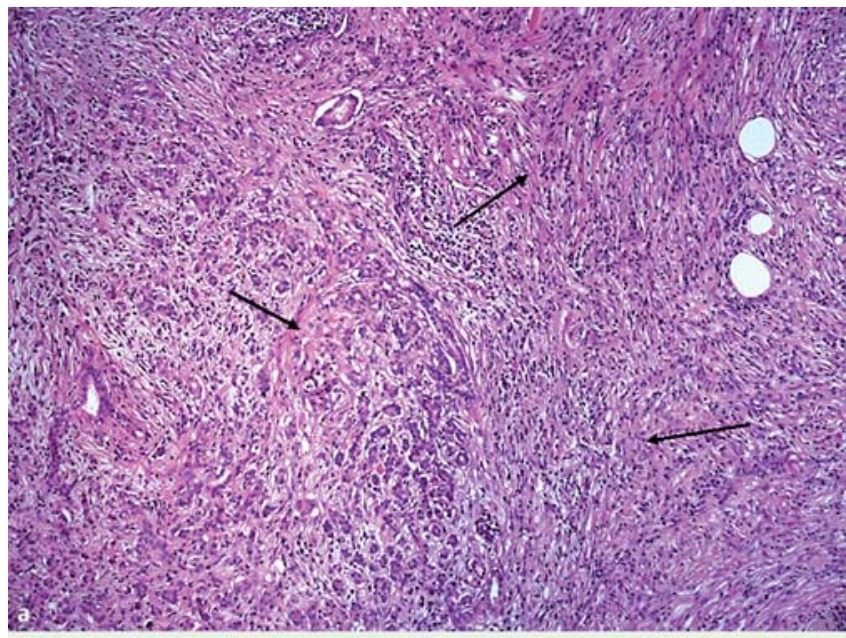

Fig. 3 Histological re-examination of the surgical specimen showing: a typical signs of autoimmune pancreatitis including lymphoplasmacytic infiltration with storiform fibrosis (arrows) on hematoxylin and eosin (H\&E) staining; b cells that are positive for IgG4 (dark brown staining).

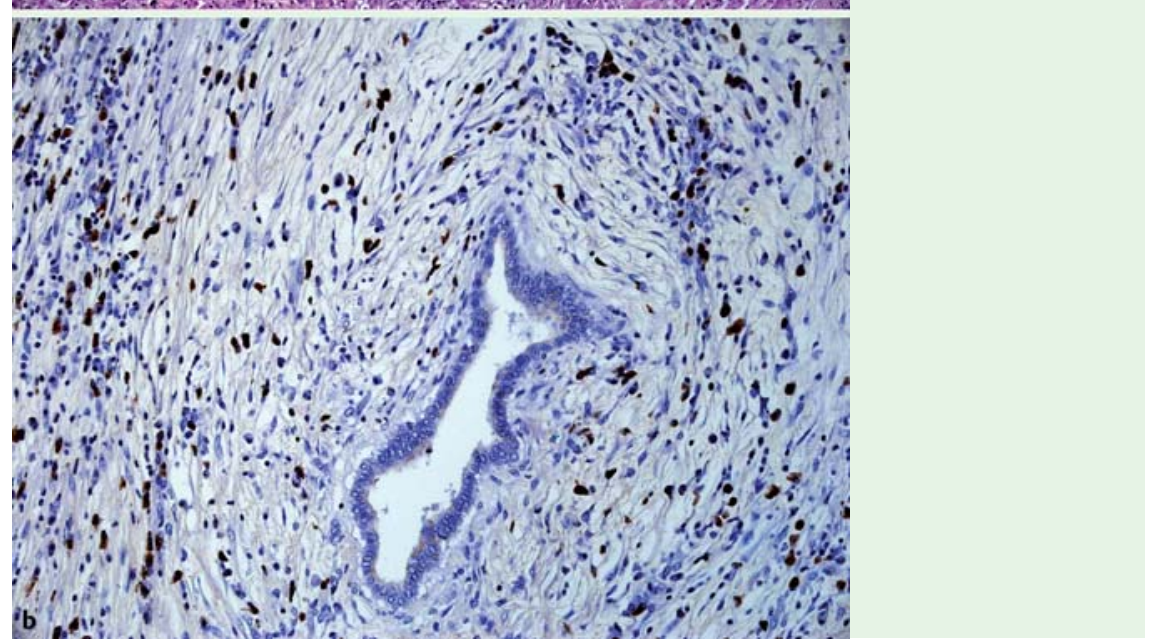

\section{References}

1 Sah RP, Pannala R, Zhang $L$ et al. Eosinophilia and allergic disorders in autoimmune pancreatitis. Am J Gastroenterol 2010; 105: $2485-2491$

2 Otsuki M, Chung JB, Okazaki K et al. Asian diagnostic criteria for autoimmune pancreatitis: consensus of the Japan-Korea Symposium on Autoimmune Pancreatitis. J Gastroenterol 2008; 43: 403-408

3 Chari ST, Smyrk TC, Levy MJ et al. Diagnosis of autoimmune pancreatitis: the Mayo Clinic experience. Clin Gastroenterol Hepatol 2006; 4: 1010-1016

4 Maillette de Buy Wenninger L, Rauws EA, Beuers $U$. What an endoscopist should know about immunoglobulin-G4-associated disease of the pancreas and biliary tree. Endoscopy 2012; 44: 66-73

5 Ghazale A, Chari ST, Smyrk TC et al. Value of serum IgG4 in the diagnosis of autoimmune pancreatitis and in distinguishing it from pancreatic cancer. Am J Gastroenterol 2007; 102: $1646-1653$

\section{Bibliography}

Dol http://dx.doi.org/

10.1055/s-0032-1309350

Endoscopy 2012; 44: E226-E227

(c) Georg Thieme Verlag KG

Stuttgart · New York

ISSN 0013-726X

\section{Corresponding author}

\section{H.-M. Curth, MD}

Department of Gastroenterology and Hepatology University of Cologne

Kerpener Str. 62

50924 Cologne

Germany

Fax: +49-221-4786758

harald-morten.curth@uk-koeln.de 